\title{
KHAYELITSHA 2001 - 2011: 10 YEARS OF PRIMARY CARE HIV AND TB PROGRAMMES
}

\author{
Daniela Belen Garone ${ }^{1,2}, M D, P h D$ \\ Katherine Hilderbrand ${ }^{\mathbf{1 , 2}}, B S c, M S c$ \\ Andrew M Boulle ${ }^{2}$, MB ChB, $M S c$, FCPHM, $P h D$ \\ David Coetzee $^{2}, M B C h B, M S c, F C P H M$ \\ Eric Goemaere $^{1,2}, M D, D S c$ \\ Gilles Van Cutsem, ${ }^{1,2} M D, D T M ぬ H, M P H$ \\ Donela Besada $^{\mathbf{1}, \mathbf{2}}, B S c, M P H$ \\ ${ }^{1}$ Médecins Sans Frontières, Khayelitsha, Cape Town, South Africa \\ ${ }^{2}$ Centre for Infectious Disease Epidemiology and Research, School of Public Health and Family Medicine, Faculty of Health Sciences, \\ University of Cape Town
}

Tuberculosis (TB) and HIV care in Khayelitsha, and in South Africa as a whole, has overcome numerous obstacles in the past three decades. This article highlights what has been achieved in Khayelitsha, describes the key clinical programme and policy changes that have supported universal coverage for HIV and TB care over the last 10 years, and outlines the challenges for the next decade.

The evolution of tuberculosis (TB) and HIV care in Khayelitsha, and in Africa as a whole, has overcome numerous obstacles in the past three decades: poor leadership in acknowledging the HIV crisis, inadequate provision of appropriate scientific interventions, and scepticism about the feasibility of treatment programmes in settings challenged with extreme resource constraints. Over the past 10 years in Khayelitsha, HIV has been transformed from less than 500 people tested for HIV and no one on antiretroviral therapy (ART) in 1998 to 50 000 tested and 20000 on ART in 2011. ${ }^{1}$ Stakeholders in the Khayelitsha sub-district have reflected in the course of the past year on the previous decade of service developments as part of commemorating 10 years of public sector ART provision. This article highlights what has been achieved collectively by several service providers (the City of Cape Town, Médecins Sans Frontières, the Western Cape province, academic institutions, the Treatment Action Campaign (TAC), nongovernmental and community-based organisations), describes the key clinical programme and policy changes that have supported universal coverage for HIV and TB care over the past 10 years, and outlines the challenges for the next decade.

\section{HIV PREVENTION AND INCIDENCE REDUCTION}

Dedicated efforts have been made to scale up a combination of prevention interventions that has resulted in substantial changes in health outcomes over the past decade.

\section{PREVENTION OF MOTHER-TO-CHILD TRANSMISSION OF HIV}

The implementation of a prevention of mother-tochild transmission (PMTCT) programme in 1999, with antenatal and perinatal antiretroviral chemoprophylaxis and free exclusive formula feeding, marked the beginning of public sector antiretroviral-based services in South Africa. This programme, with progressive improvements and intensification in line with the evolution of national guidelines, has resulted in a reduction in documented mother-to-child transmission from $12.5 \%$ in 2002 to $2.5 \%$ in 2010 based on polymerase chain reaction (PCR) testing at 6 weeks (Fig. 1). This success is mirrored in many other sites in South Africa, and demonstrates that PMTCT to very low proportions of infants infected is both feasible and scalable.

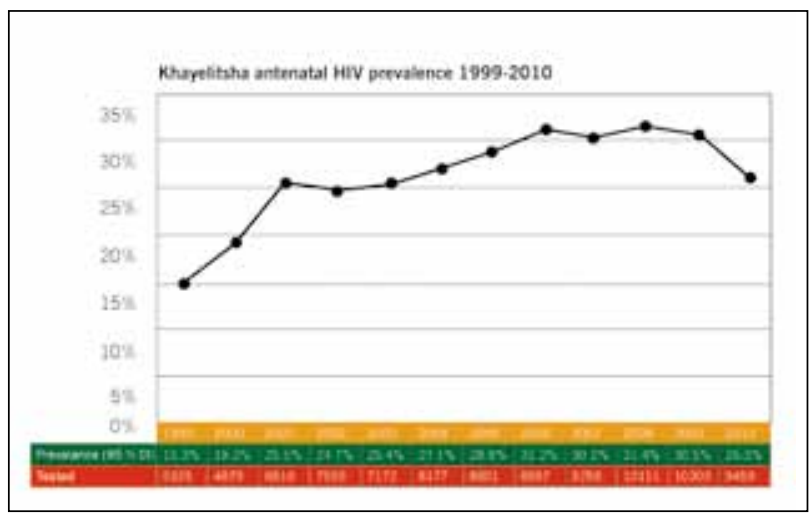

Fig. 1. Rate of mother-to-child HIV transmission, 2002 - 2010. 


\section{Box 1. Key elements of the programme}

Khayelitsha is a large township with approximately 500000 inhabitants, located on the outskirts of Cape Town. It has one of the highest burdens of both HIV and toberculosis (TB) in South Africa. An estimated $16 \%$ of the population is HIV infected; TB incidence is above $1500 / 100000$ per year and TB/HIV co-infection is close to $70 \%$. The incidence of drug-resistant TB is estimated at $50 / 100000$ per year. ${ }^{11}$

The Khayelitsha programme, started in 1999, was the first in South Africa to provide antiretroviral therapy (ART) at primary care level in the public sector and one of two pilot projects in the country to provide decentralised care for drug-resistant tuberculosis. ${ }^{12}$ Key strategies implemented include:

- prevention of mother to child transmission with ART and formula

- large-scale HIV counselling and testing, including out-of-facility testing, youth clinics, and male clinics

- mass community condom distribution

- decentralisation of ART to all clinics in the subdistrict

- 'one-stop-shop' integration of ART and TB services

- nurse management of HIV and TB care, including nurse-initiated ART and TB treatment

- doctor support and mentorship, with a strong secondary care referral system

- district level planning and co-ordination

- three-tier monitoring and evaluation system (paper register, electronic register,and electronic medical record in selected sites)

- ongoing training and mentoring at clinic and district level

Furthermore, the decentralisation of paediatric ART into primary care clinics, which started in 2004, resulted in a steady increase in children being initiated on ART each year, from 4 in 2001 to 145 in 2008 . The numbers have decreased since then to 115 in 2010 . The successful decentralisation of paediatric care has led to positive health outcomes; $87 \%$ of children started on ART in primary care remained in care, and $98 \%$ remained alive after 5 years on ART.

\section{HIV TESTING}

HIV testing has increased steadily since the advent of the programme. The programme started with enzyme-linked immunosorbent assay (ELISA) testing of 500 people in January 1999. The introduction of rapid HIV testing kits, PCR testing for infants, employment of lay counsellors to conduct HIV counselling and testing (HCT), communitybased testing sites, wide-scale HCT campaigns, and targeted testing for TB patients/suspects, youth and males saw a dramatic increase in the numbers of people being tested each year. By the end of 2010, 22 centres were providing HCT and approximately 57000 people were being tested annually. In a community survey conducted in 2004, $28 \%$ of men and $53 \%$ of women of reproductive age in Khayelitsha reported having been tested for HIV. ${ }^{2}$

\section{COMMUNITY CONDOM DISTRIBUTION}

Male condom distribution has been a major priority in the programme. The number of condoms distributed per year increased from 2 million in 2004 to more than 10 million in 2006; currently more than a million condoms are distributed every month. This was made possible through the combined efforts of the public sector, including the City of Cape Town, and non-governmental organisations, especially TAC. TAC expanded condom distribution from health care facilities to community distribution points, such as taxi ranks, public libraries, toilets and shebeens. ${ }^{3}$ During the same period, the number of adults reported to have been treated for sexually transmitted infections (STIs) decreased fourfold, from 28000 in 2004 to less than 5000 in 2009.

\section{OPENING OF A MALE-FRIENDLY CLINIC}

Recognition of the need for a service dedicated to reaching men and providing $\mathrm{HCT}$ and STI treatment resulted in the opening of a male walk-in clinic at a taxi rank in 2007. This new service was widely advertised in the community through the use of taxi ranks and the local radio. This clinic has become the largest STI treatment site in the Cape Metro area, with the number of STls treated increasing from 843 in 2007 to 2547 in 2010. In the first half of 2011 the clinic counselled and tested as many men as were tested throughout 2010, and the number tested during 2010 in the male clinic represented $27 \%$ of all men tested in Khayelitsha. The clinic aims to promote and empower men to take ownership of their sexual health and safety and that of their partners. Its success demonstrates that men use health services that are adapted to their needs: short waiting times, close to a usual gathering place for men (taxi rank), and separate from public health services attended mostly by women and children. Two new male clinics are planned for 2011 to continue to reach out to men, who remain a group with limited access to care.

\section{ANTIRETROVIRAL THERAPY}

In May 2011 there were 20000 patients on ART, which is estimated to represent $63 \%$ coverage of those in need in Khayelitsha, according to current World Health Organization (WHO) eligibility criteria. Retention in care at one year on ART has remained consistently above $85 \%$ since the beginning of the programme. ${ }^{4}$ In addition, virological suppression among patients in care also remained above $87 \%$ in those tested, ${ }^{4}$ thereby decreasing the community viral load given the relatively high proportion of infected adults who are on ART. It is therefore likely that this is contributing to a reduction in the number of new HIV infections.

\section{EVOLUTION OF HIV PREVALENCE}

HIV prevalence among women presenting for antenatal care has been routinely measured by the programme since 1999, and since 2003 the testing acceptance rate has been close to $100 \%$. The antenatal HIV prevalence among those who test in routine care has stabilised since 2006 and may now be declining (Fig. 2). This decline is not mirrored by the annual anonymous antenatal survey, which might be explained by the fact that in routine care 
women already on ART are often not retested for HIV, whereas during the annual survey all pregnant women are tested, regardless of whether they are on ART or not. Considering the decreased mortality, the absence of an increase in antenatal prevalence in recent years could be the result of the benefits of ART offsetting reduced new infections.

Emerging data illustrate that treatment serves as a powerful prevention tool, ${ }^{5}$ and to decrease HIV incidence there is a need to combine wide-scale access to ART with available prevention tools, including HCT, PMTCT and condom distribution. In parallel, there is the need to focus on innovative tools to measure HIV incidence in order to adequately assess HIV prevention efforts.

\section{DECREASING MORBIDITY}

Increasing access to ART, as a result of decentralisation, task shifting and TB/HIV integration, has allowed patients to access and initiate treatment earlier. This in turn has resulted in decreasing HIV-related mortality and morbidity. Patients presenting to care earlier are also typically less complex to manage clinically, which facilitates increased nurse management of care.

\section{EVIDENCE OF EARLIER ART ACCESS}

Median CD4 counts of people starting ART increased from 43 cells $/ \mu$ in 2001 to $162 \mathrm{cells} / \mu \mathrm{l}$ in 2010. In the same period the proportion of patients presenting with a WHO stage IV diagnosis decreased from $50 \%$ to $20 \%$.

\section{BETTER ART REGIMENS}

The programme has also seen a gradual evolution towards improved first-line regimens. Documentation of high rates of adverse events with the use of stavudine (d4T) contributed to the growing evidence base that led to the WHO recommendation to replace d4T with better-tolerated alternatives. ${ }^{6} \mathrm{~A}$ tenofovir-based regimen was introduced in 2005 in Khayelitsha for patients experiencing d4T toxicity, and was included in the national first-line regimen for all patients in 2010.

\section{INTEGRATION OF TB AND HIV SERVICES}

The 'one-stop-shop' integration of TB and HIV services began in 2004, and allowed co-infected patients to access TB and HIV services in the same clinic and from the same health staff. ${ }^{7}$ In $2010,99 \%$ of TB patients in TB/HIV integrated clinics were offered HCT, and $95 \%$ of

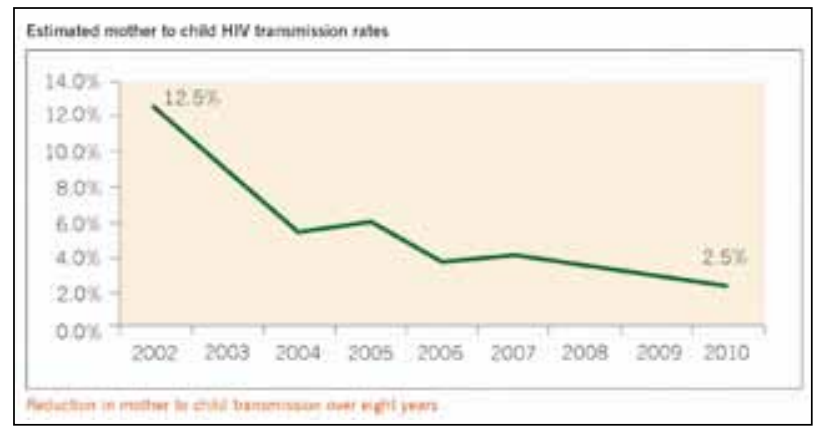

Fig. 2. Khayelitsha antenatal prevalence, $1999-2010 . \mathrm{CHC}=$ community health centre; City Clinic = City of Cape Town Clinic.
Box 2. Community-based model of care for drugresistant tuberculosis

- Drug-resistant tuberculosis (DR-TB) refers to TB that has become resistant to first-line treatment and requires longer and more difficult treatment with second-line anti-TB drugs.

- Instead of attempting to hospitalise all DR-TB patients, the Khayelitsha model of care uses a patient-centred approach with community-based treatment through existing primary care services.

- Using lessons learned from the decentralisation of HIV care, diagnosis and treatment of DR-TB has been integrated into the routine TB and HIV programmes in Khayelitsha since early 2008.

them were being tested. Furthermore, $99 \%$ of co-infected patients had a CD4 count result recorded and over $95 \%$ were started on co-trimoxazole preventive therapy. The successful integration of the services has led to improvements in the detection rates of smear-negative pulmonary TB and extrapulmonary TB, as nurses' clinical skills in managing both diseases improved. In addition, integration has resulted in a decrease in the median time from the start of TB treatment to ART initiation. ${ }^{7}$ Improved TB diagnostic methods, including smear-negative algorithms, systematic TB culture, lineprobe assays, and the piloting of GeneXpert, as well as the systematic screening of HIV patients for TB, are all expected to contribute to increased TB diagnosis. The integration of HIV/TB care has been accompanied by improved TB cure rates (from $44 \%$ in 2005 to $81 \%$ in 2010), despite the increased caseload.

\section{COMMUNITY-BASED MANAGEMENT OF DRUG-RESISTANT TB}

Before 2007, all patients with drug-resistant tuberculosis (DR-TB) in Khayelitsha had to be admitted to a central TB hospital to receive their treatment. Khayelitsha has piloted a community-based DR-TB programme in which drugresistant TB is diagnosed and treated in primary care clinics as opposed to a centralised facility. The number of cases of DR-TB diagnosed increased from 14 in 2003 to 200 in 2010 . Over $80 \%$ of patients diagnosed with DR-TB in 2009 and 2010 started treatment. The decentralisation of care was able to ensure that $71 \%$ of cases started treatment through the local clinic, while only $14 \%$ were admitted to the centralised specialist DR-TB hospital. In addition, the median time to treatment initiation decreased from 71 days in 2007 to 33 days in 2010 .

\section{DECREASING MORTALITY \\ DECENTRALISED, TB/HIV INTEGRATED, NURSE-MANAGED ART}

Recorded mortality of adults at 3 months on ART decreased from $10 \%$ in 2002 to $2.2 \%$ in 2010 , in part due to patients accessing ART earlier. While mortality ascertainment is less complete in recent years, there remains a year-on-year decline in mortality even after linkage to the national death registry to correct for mortality under-ascertainment. ${ }^{4}$ Recorded mortality of adults at one year on ART decreased from under $15 \%$ to $8 \%$ between 2002 and $2007 .{ }^{4}$ This decline in 
early mortality and earlier access to ART has occurred alongside a dramatic increase from 100 people initiated on ART in 2001 to approximately 20000 by mid-2011 (Fig. 3). The sharp rise in treatment enrolment was made possible due to decentralisation of ART to every clinic, TB/HIV integration, and nurse-managed ART and TB care. Decentralised care began in 2006 and new clinics were accredited as ART sites each year thereafter. National guidelines allowed for nurse-initiated TB/ HIV integrated ART in April 2010, and all 11 clinics in Khayelitsha were providing ART by the end of 2010. In terms of longer-term outcomes, previous data based on death registry linkage have also confirmed that 4 out of 5 patients who started treatment were alive 5 years after starting treatment. ${ }^{4}$

\section{PATIENTS TREATED FOR DRUG-RESISTANT TUBERCULOSIS}

While there has been an increase in the detection of DR-TB, the Khayelitsha programme has seen an improvement in the survival of people with DR-TB. Of those diagnosed with DR-TB in 2008, $62.4 \%$ remained alive after 18 months, reflecting an overall mortality of $38 \%$. While mortality levels remain high, given that $76 \%$ of all DR-TB patients are HIV infected, this represents significant improvements in health outcomes among people with DR-TB compared with other settings. ${ }^{8}$

\section{CHALLENGES RETENTION IN CARE}

While the programme has achieved many successes, several challenges remain in ensuring universal access to ART. With the advent of improved drug regimens and models of care, HIV has become a manageable chronic disease. Approximately $85 \%$ of patients are retained in care after 12 months, while only $65 \%$ of patients are still in care at 5 years on treatment; this highlights the need for further innovations to improve long-term retention. With increased enrolment came increased losses to follow-up, probably due to saturation of services, patient mobility and death. This trend in patient losses to followup stabilised and even began to decrease in the later years as a result of the adoption of measures to adapt to the high numbers of patients on treatment. Innovative models of care included the introduction of adherence clubs and nurse management of patients.

\section{MEN}

The proportion of men among adults starting ART remains lower than expected. Although the male walk-

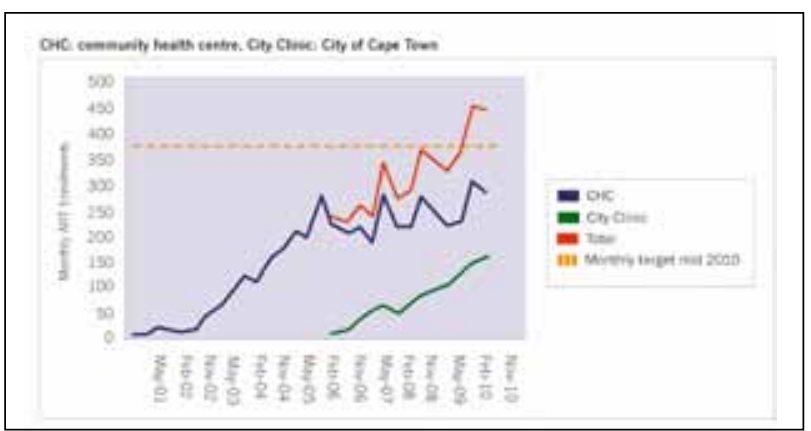

Fig. 3. Monthly patient ART enrollment, 2001 - 2010. in clinic has driven an increase in men testing for HIV and seeking treatment for STIs, men still represent only $30 \%$ of adults in care after 10 years of ART treatment in Khayelitsha. There is a need to develop programmes that cater to men's needs in order to improve their access to health services. Furthermore, given that men rarely accompany their partners to antenatal services, innovative mechanisms will be required to identify serodiscordant couples in light of emerging guidelines that will recommend the use of ART to reduce the risk of HIV transmission to the negative partner. ${ }^{5}$

\section{YOUTH}

Pre-ART loss to follow up is particularly high among youth. In 2010 , up to $70 \%$ of eligible young people in the youth clinics were lost to care before starting ART; $60 \%$ of these losses occurred immediately after HIV testing. Youth on ART were also more likely than older adults to be lost to follow-up. ${ }^{9}$ Recognition of the need to focus specific interventions targeted at this high-risk group led to the establishment in 2005 of two youth clinics that provide adapted and targeted services. Although pre-ART loss to follow-up declined in 2010 - from $70 \%$ and $60 \%$ in the first quarter to $45 \%$ and $29 \%$ in the last quarter for the two youth clinics, respectively - early loss to care remains high and is indicative of the need to continue to adapt services and interventions that cater appropriately to the needs of youth.

\section{TREATMENT FAILURE}

At 5 years on ART, approximately $14 \%$ of patients had confirmed virological failure and $12 \%$ were on secondline ART. ${ }^{4}$ Mortality and treatment failure are found to be high in patients on second-line treatment. Out of the $32 \%$ of patients who failed second-line ART, $60 \%$ had poor adherence, and $30 \%$ returned to undetectable viral loads after enhanced adherence support; the remainder were switched to a third-line regimen. This highlights the need for early detection of poor adherence in order to provide targeted enhanced adherence support. A recent study in Khayelitsha demonstrated that a viral load performed at 3 months resulted in better virological outcomes than one performed at 6 months, ${ }^{10}$ demonstrating the usefulness of the viral load for early detection of poor adherence and virological failure. Third-line drugs are currently not available in the public sector due to their high costs. As access to and time on ART increases, the number of patients requiring second- and third-line regimens is rising, and prices for these drugs will need to be driven down.

\section{THE FUTURE}

The key clinical challenges for the next decade will be to achieve universal coverage of patients in need of ART, retain patients in care, and decrease the number of new infections. This will necessitate innovative models of care to decrease the burden of patients on chronic treatment on health facilities - thereby allowing the facilities to increase enrolment on ART - and improve long-term adherence and retention, as well as the implementation of combination prevention strategies. 
Box 3. Strategies for the future - innovation and wide implementation

- Further out-of-facility community-based testing (in schools, taxi ranks, community halls)

- Initiation of antiretroviral therapy at higher CD4 thresholds, potentially up to CD4s of $500 \mathrm{cells} / \mu \mathrm{l}$ and/or high viral load thresholds, to further reduce viral circulation and infectiousness at community level (treatment as prevention, TASP)

- Community-based delivery of ART by community health workers and/or chronic dispensing units

- Increased investment in pre-ART (e.g. by creating pre-ART adherence clubs)

- New pre-exposure prophylaxis strategies targeting young female adolescents combined with development of new long-acting antiretroviral formulations and other biomedical preventive interventions.

These strategies will have to be supported by new drug formulations and technologies, including:

- fixed-dose combinations for first- and second-line regimens

- semi-quantitative point of care viral load dipstick to monitor adherence and identify early treatment failures

-POC CD4 devices to reduce pre-ART loss to followup, mostly among adolescents

- more robust regimens with a better safety profile, including drugs like darunavir, which maintains a low toxicity in the long term once the viral load has become undetectable

- ensure appropriate access to reproductive health services, including Pap smears for all women including those living with HIV and medical termination of pregnancy at the primary health level.

\section{ADHERENCE CLUBS}

Adherence clubs were established to improve clinic efficiency (decongest clinics and allow clinicians time for initiating patients), sustain high enrolment targets, and improve long-term adherence. The adherence clubs are group clinic visits for stable patients that are run by lay health workers and meet every 2 months. On club days patients receive their medication and are screened for opportunistic infections and adverse events, and are referred to a clinician if necessary. In addition, patients receive an educational talk on the day of their visit.

By the end of 2010 , a total of 30 clubs were created, 23 of them situated in the community and 7 in the Ubuntu Clinic in Khayelitsha. More than 750 people were enrolled in total. An early evaluation revealed that after 1 year of enrolment in the clubs, $99.2 \%$ of the patients were alive; at 2 years this figure stood at $97.5 \%$. After the first year of enrolment, loss to follow-up was $1.1 \%$ and mortality $0.7 \%$. This model is currently being expanded to other clinics in order to increase coverage. With the adoption of new national regulations, community health care workers will now be able to manage these clubs effectively. There is still a need to adapt the drug distribution system further in order to allow for regulated and quality-assured chronic drug dispensing units that are able to provide medications more conveniently to patients in the community, thereby ensuring that there is increased access to treatment closer to patient's homes.

\section{REDUCING HIV AND TB INCIDENCE}

Despite some early positive signs, the national target of a $50 \%$ reduction in HIV incidence is not close to being achieved. An exception to this is in the area of PMTCT, where transmission has been reduced by $80 \%$ since the beginning of the programme. There is a need to continue to focus on sustained behavioural interventions, including widespread condom distribution, out-of-facility community-based testing, and biomedical interventions to reduce HIV incidence. New targeted pre-exposure prophylaxis strategies (particularly for women wishing to have children), initiation of ART at a CD4 count of 500 cells/ $\mu$, new drug formulations and technologies including fixed-dose combinations, point-of-care viral load and CD4 testing, and robust low-toxicity regimens must be explored.

Furthermore, there is a need to continue to focus on the early diagnosis and treatment of TB and DR-TB, both to improve individual patient outcomes and to reduce transmission. Despite dramatic improvements in case detection for DR-TB, only half of all estimated DR-TB cases are diagnosed in Khayelitsha, and there is a need to increase case detection to curb HIV transmission. Access to molecular diagnostics, including GeneXpert, shows promising results in increasing detection of TB and DR-TB. This could also provide a sensitive screening tool for the provision of INH prophylaxis in TB-negative patients.

\section{DISCUSSION}

The success in scaling up HIV/TB service provision in Khayelitsha is attributed to the collaborative efforts of service providers, policy makers, academics, civil society and the community at large. The Khayelitsha programme was successful in achieving community buy-in because it offered a reliable service within the community, was supported through partnerships, and was complemented by widespread treatment literacy.

While the district is best known for its role in demonstrating the feasibility of ART in resource-constrained settings, some of the most important lessons have come in more recent years, where the latent capacity of South Africa's public health system has been demonstrated when subjected to energy, innovation and meaningful collaboration. In spite of the numerous partnerships, Khayelitsha remains a difficult environment in which to deliver services, but it has nevertheless been possible to achieve coverage and scale for a number of activities beyond the most optimistic outlooks of a decade ago. In the areas of PMTCT and ART provision this is being demonstrated in South Africa as a whole, where expectations have been exceeded in recent years.

Increased funding for antiretrovirals and the resulting increase in access to care helped to strengthen the 
overall health system, and the implementation of a large-scale TB/HIV programme resulted in decreased rates of both illness and death among people living with HIV as well as a likely reduction in the number of new HIV infections. This unique clinical programme contributed to national policy changes that have had a tangible impact on the lives of thousands of people living with HIV and TB in South Africa, and has demonstrated the possibility of achieving universal coverage of ART and positive patient outcomes in resource constrained settings.

Challenges ahead include the need to reduce HIV transmission in the community. The Khayelitsha programme has paved the way for innovative approaches to treatment provision that have allowed an increasing number of people to access quality treatment closer to their communities. The future of HIV and TB treatment and care will require a focus on combination prevention and treatment interventions, in addition to the adoption of new innovations that can have a tangible impact on the spread of the dual epidemics.

\section{REFERENCES}

1. Médecins Sans Frontières. Khayelitsha 2001-2011 activity report: 10 years of HIV/ TB care at primary health care level. June 2011. HYPERLINK "http://www.msf.org. za/publication/khayelitsha-activity-report-2001-2011-10-years-hivtb-care-primaryhealth-care-level" http://www.msf.org.za/publication/khayelitsha-activity-report- 2001-2011-10-years-hivtb-care-primary-health-care-level (accessed 11 August 2011)

2. Boulle A, Hilderbrand K, Menten J, et al. Exploring HIV risk perception and behaviour in the context of antiretroviral treatment: results from a township household survey. AIDS Care 2008;20(7):771-781

3. South African Department of Health. A million condoms a month. 2010. HYPERLINK "http://www.doh.gov.za/docs/hiv/million-condoms.pdf" http://www.doh.gov.za/docs/ hiv/million-condoms.pdf (accessed 11 August 2011).

4. Boulle A, Van Cutsem G, Hildebrand K, et al. Seven-year experience of a primary care antiretroviral treatment programme in Khayelitsha, South Africa. AIDS 2010;24(4):563-572.

5. Cohen MS, Chen YQ, McCauley M, et al. Prevention of HIV-1 infection with early antiretroviral therapy. N Engl J Med 2011;365(6):493-505.

6. Boulle A, Orrel C, Kaplan R, et al. Substitutions due to antiretroviral toxicity or contraindication in the first 3 years of antiretroviral therapy in a large South African cohort. Antivir Ther 2007;12(5):753-760.

7. Brown C, Kerschberger B, Boulle A, et al. TB and HIV service integration within a South African primary health care setting reduces the time to ART initiation without negatively impacting TB outcomes. Presented at the 18th Conference on Retroviruses and Opportunistic Infections (CROI 2011), 27 February - 2 March 2011, Boston, USA. Abstract 890. HYPERLINK "http://www.retroconference.org/2011/ Abstracts/41110.htm"http://www.retroconference.org/2011/Abstracts/41110.htm (accessed 11 August 2011)

8. Médecins Sans Frontières, Partners in Health, Treatment Action Group. An evaluation of drug-resistant TB treatment scale-up. Switzerland, July 2011. HYPERLINK "http:// www.ghdonline.org/uploads/DRTB_Report_A4_Final.pdf"http://www.ghdonline.org/ uploads/DRTB Report_A4_Final.pdf (accessed 11 August 2011).

9. Van Cutsem G, Ford N, Hildebrand K, et al. Correcting for mortality among patients lost to follow up on antiretroviral therapy in South Africa: a cohort analysis. PLoS One 2011;6(2):e14684

10. Kerschberger B, Boulle A, Hilderbrand K, Coetzee D, Goemaere E, Van Cutsem G Viral load at 3 months associated with better virologic outcomes than at 6 months. Presented at the 6th IAS Conference, 17-20 July 2011, Rome. MOPE144. http://pag. ias2011.org/Abstracts.aspx?AID=3320 (accessed 11 August 2011).

11. Cox HS, McDermid C. Azevedo V, et al. Epidemic levels of drug resistant tuberculosis (MDR and XDR-TB) in a high HIV prevalence setting in Khayelitsha, South Africa. PLoS One 2010;5(11):e13901.

12. Médecins Sans Frontières. Scaling up diagnosis and treatment of drugresistant tuberculosis in Khayelitsha, South Africa. An integrated, communitybased approach. March 2011. HYPERLINK "http://www.msf.org.za/system/files/ publication/documents/DR-TB-in-Khayelitsha-Mar2011. pdf?download=1"http://www. msf.org.za/system/files/publication/documents/DR-TB-in-Khayelitsha-Mar2011. pdf?download=1 (accessed 11 August 2011).

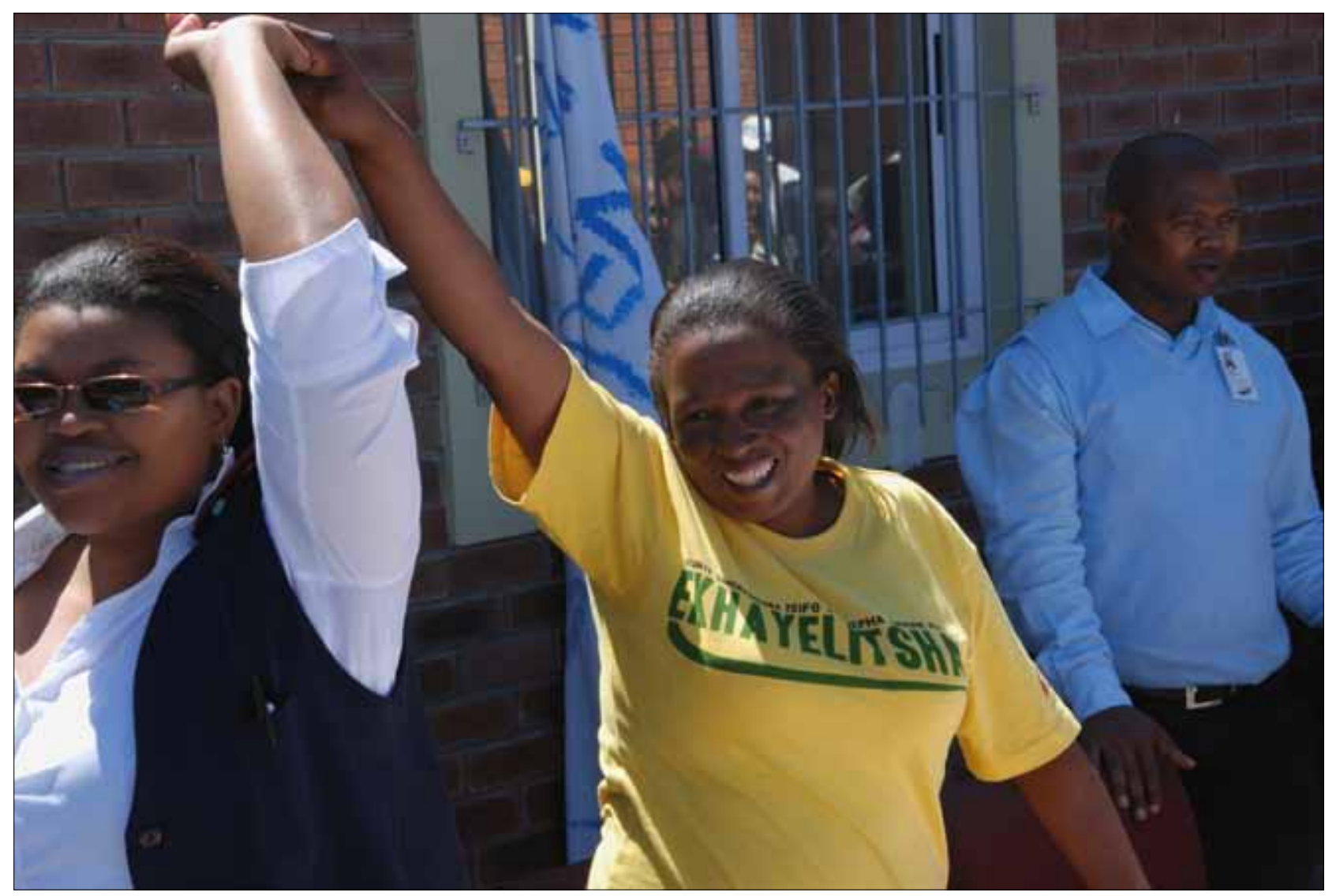

